\title{
Chaos in Self-Oscillatory Circuits Containing Time Delay
}

Takashi Maruyama, Naohiko Inabał, Yoshifumi Nishio and Shinsaku Mori Department of Electrical Engineering Keio University 3-14-1 Hiyoshi, Kohoku-ku, Yokohama, 223 Japan

$\nmid$ Department of Information Science Utsunomiya University

2753 Ishii-machi, Utsunomiya, 321 Japan

\begin{abstract}
This paper gives rigorous analysis of chaos in two types of self-oscillatory circuits containing time delay. The governing equation of each circuit is described by 2 nd-order differential equation containing time delay. By using mapping method, we can derive the 1-dimensional Poincaré map explicitly from each circuit, and the Poincare map is proved to have a positive Liapunov number with computer assistances. Moreover the proposed circuits are realized easily so that the theoretical results can be verified experimentally.
\end{abstract}

\section{INTRODUCTION}

Many self-oscillatory systems containing time delay have been studied. Recently, chaotic phenomena are observed in such systems[1] [3]. For example, Ueda et al.[2] and Kouda et al.[3] have investigated the Minorsky's system in detail. The Minorsky's system is an example of self-oscillatory systems, and the governing equation (the Minorsky's equation) is described by the nonlinear difference-differential equation. It exhibits chaos when that system has the large time delay[2] or the higher-order nonlinear characteristics[3], and these results are verified by the computer simulations. However the theoretical evidence of chaos has not yet been clarified, since it is very difficult to solve the nonlinear differencedifferential equation strictly.

On the other hand, 1-dimensional mappings are mathematically studied in detail. For example, the sufficient condition where the mapping has a positive Liapunov number is given by Lasota, Li and Yorke[4][5].

In this paper, we propose two types of self-oscillatory circuits containing time delay which exhibit chaos, and analyze them strictly. The governing equation of each circuit is described by 2 nd-order differential equation containing time delay. By using mapping method, the Poincaré map can be derived explicitly as a 1-dimensional map from each circuit. Furthermore we obtain the chaos generating condition where the mapping has a positive Liapunov number, and show the chaos generating region in parameter space by computer calculations. Lastly the theoretical results are verified by circuit experiments.

\section{CIRCUIT MODELS}

Fig. 1 shows the circuit models. In this figure, $-g$ is a linear negative conductance, and each constant is selected so that $v$ can vibrate. Each circuit consists of a $-g L C$ oscillator and a loop which controls an amplitude of circuit.

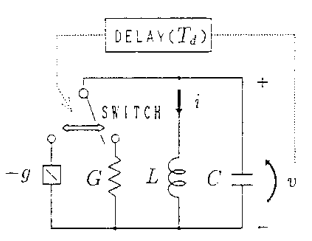

(1) Circuit-1

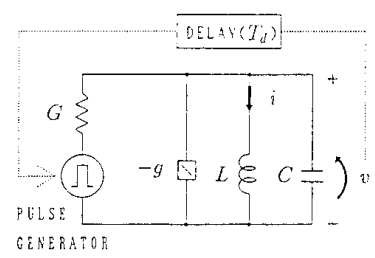

(2) Circuit-2
Fig.1 Circuit models

Fig.2 shows the switching operation and the pulse generating operation.

(a) if $v<V_{t h}$

the switch is connected to $-g$ (circuit-1)

the pulse is not added (circuit-2)

(b) if $v \geq V_{t h}$

the switch is connected to $G$ (circuit-1)

the pulse $(V)$ is added (circuit-2)

In the case where time delay $\left(T_{d}\right)$ is contained in this operation, chaotic phenomena are generated in each circuit.

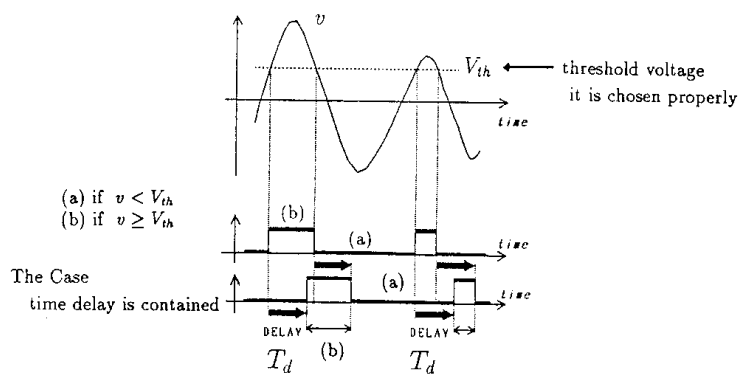

Fig.2 Switching operation (Circuit-1) and pulse generating operation (Circuit-2)

CH2819-1/90/0000-1055\$01.00@1991 IEEE 
The normalized equation of the circuit 1 is represented as follows:

$$
\ddot{x}+f\left(\dot{x}\left(\tau-\tau_{d}\right)\right) \dot{x}+x=0
$$

and $f(u)$ is switching operation and is represented by

$$
f(u)= \begin{cases}2 \delta_{2} & \text { if } u>1 \\ -2 \delta_{1} & \text { otherwise }\end{cases}
$$

where

$$
\begin{gathered}
\eta, "=\frac{d}{d \tau}, \quad i=\sqrt{\frac{C}{L}} V_{t h} \cdot x, \quad t=\sqrt{L C} \tau \\
\frac{T_{d}}{\sqrt{L C}}=\tau_{d}, \quad \sqrt{\frac{L}{C}} g=2 \delta_{1}, \quad \sqrt{\frac{L}{C}} G=2 \delta_{2} .
\end{gathered}
$$

On the other hand, the normalized equation of the circuit2 is represented as follows:

$$
\ddot{x}-2 \delta \dot{x}+x=f\left(\dot{x}\left(\tau-\tau_{d}\right)\right)
$$

and $f(u)$ is pulse generating operation and is represented by

$$
f(u)= \begin{cases}\alpha & \text { if } u>1 \\ 0 & \text { otherwise }\end{cases}
$$

where

$$
\sqrt{\frac{L}{C}}(G-g)=-2 \delta, \quad V \cdot G=\alpha .
$$

Fig. 3 shows an example of the chaotic attractor generated in each circuit.

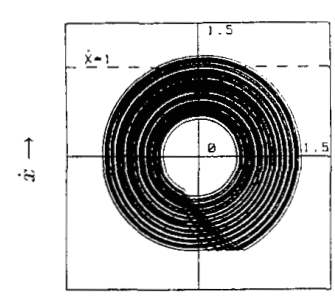

(1) Circuit-1

$\left(\delta_{1}=0.02, \delta_{2}=0.6, \omega_{1} \tau_{d}=\pi\right)$

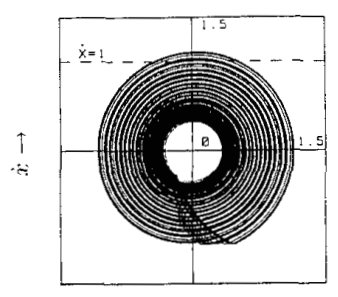

(2) Circuit-2 $\left(\delta_{1}=0.02, \alpha=1, \omega_{1} \tau_{d}=\pi\right)$
Fig.3 Chaotic attractor

\section{DERIVATION OF POINCARÉ MAP AND ITS ANALYSIS}

The Poincaré map of each circuit can be derived explicitly as a 1-dimensional map. In this section, we explain the derivation of the Poincaré map and its analysis by using the circuit-1.

Fig. 4 shows the vector field on the $x-\dot{x}$ plain.

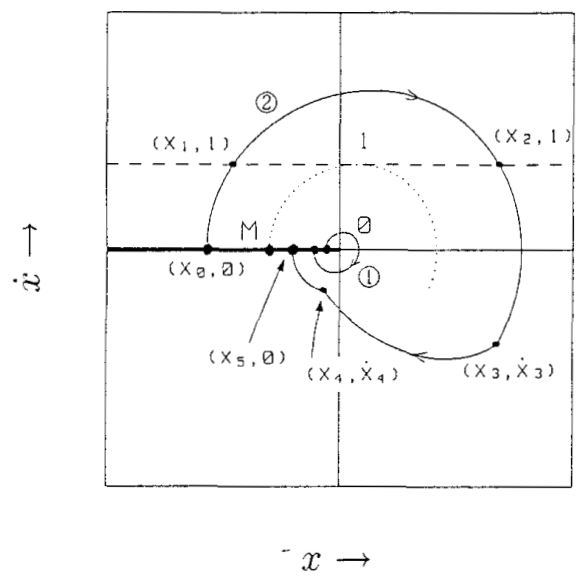

Fig.4 Vector field on the $x-\dot{x}$ plain

As an initial condition, the following case is considered:

$$
(\tau, x, \dot{x})=\left(0, x_{0}, 0\right)
$$

We define $L$ and $L_{t h}$ as

$$
\begin{gathered}
L=\{x, \dot{x} \mid x<0, \dot{x}=0\} \\
L_{t h}=\{x, \dot{x} \mid \dot{x}=1\} .
\end{gathered}
$$

Furthermore let $M$ be the value of $x$ at an initial point on $L$ whose flow should be tangent to the line $L_{t h} . M$ is represented explicitly as

$$
M=-\exp \left\{-\frac{\delta_{1}}{\omega_{1}}(\pi-\theta)\right\} \text { where } \theta=\arctan \frac{\omega_{1}}{\delta_{1}} .
$$

Now we discuss the solution whose initial condition is represented by Eq.(3). The following movements of the solutions are divided into two cases (I) and (II).

(I) Case $M<x_{0}<0$ ( (1) in Fig.4)

The solution crosses $L$ again at $\tau=2 \pi / \omega_{1}$ without reaching the threshold $L_{t h}$.

(II) Case $x_{0}<M$ ( (2) in Fig.4)

The solution starting from $L$ crosses $L_{t h}$ at $(\tau, x, \dot{x})=$ $\left(\tau_{1}, x_{1}, 1\right)$. The solution continues to rotate divergently around $O$ until $\tau=\tau_{1}+\tau_{d}$. The solution crosses $L_{t h}$ again at $(\tau, x, \dot{x})=\left(\tau_{1}+\tau_{2}, x_{2}, 1\right)$. Then, the solution rotates convergently around $O$ from $(\tau, x, \dot{x})=\left(\tau_{1}+\right.$ $\left.\tau_{d}, x_{3}, \dot{x}_{3}\right)$ to $\left(\tau_{1}+\tau_{2}+\tau_{d}, x_{4}, \dot{x}_{4}\right)$.

For the sake of simplicity, we consider the case where $\left(x_{4}, \dot{x}_{4}\right)$ satisfies the following condition:

$$
x_{4}>0, \dot{x}_{4}<1 \quad \text { or } \quad x_{4}<0, \dot{x}_{4}<0
$$

When this condition is satisfied, the solution starts to rotate divergently and crosses $L$ again at $(\tau, x, \dot{x})=$ $\left(\tau_{1}+\tau_{2}+\tau_{3}+\tau_{d}, x_{5}, 0\right)$. 
Therefore, the Poincaré map $F$ can be defined as a 1dimensional map:

$$
F: L \rightarrow L, \quad x_{0} \rightarrow F\left(x_{0}\right)
$$

where $x_{0}$ is the $x$-coordinate at the initial value on $L$ and $F\left(x_{0}\right)$ is the $x$-coordinate at the point on $L$ which the solution leaving $L$ returns back. $F$ can be represented explicitly. However the concrete representation of $F$ is omitted due to space restrictions. Fig. 5 shows an example of $F$.

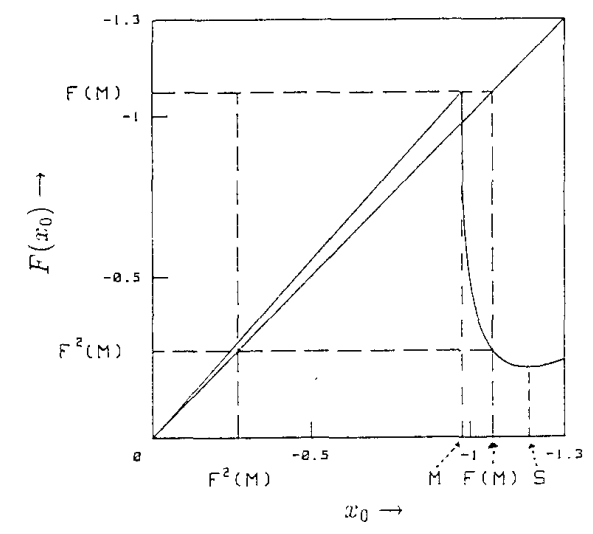

Fig.5 Poincaré map $\left(\delta_{1}=0.015, \delta_{2}=0.9, \omega_{1} \tau_{d}=\pi\right)$

In discussing about $F$, the definition is given.

[Definition 1]

An interval $J$ in $L$ is said to be invariant if $F(J) \subset J$. And the invariant interval $J$ is said to be stable if there is some interval $J^{\prime} \supset J$ and some positive integer $n$ such that $F^{n}(X) \subset J$ for all $X \in J^{\prime}$, where $F^{n}$ denotes $n$-times composite of $F$. \#

In the following discussions, we consider the case where the two extrema $M$ and $S$ satisfy the following condition (see Fig.5):

$$
F(M)>S
$$

Define

$$
J=\left[F(M), F^{2}(M)\right]
$$

If Eq.(5) is satisfied, the interval $J$ is invariant and stable, and further $F$ has a unique extremum $F(M)$ in $J$.

[Theorem 1][4][5]

We assume that $F: J \rightarrow J$. If

$$
\left|\frac{d F}{d x_{0}}\right|>1 \text { for almost all } x_{0} \in J
$$

is satisfied. It is known that $F$ has the unique absolutely continuous invariant measure and also $F$ is ergodic. $\#$

Then if Eq.(7) is satisfied, the mapping has a positive Liapunov number. Therefore, in this paper we regard that chaos is generated when Eq.(7) is satisfied.
As concerns the Poincaré map $F$, we obtain below three equations:

$$
\begin{gathered}
\lim _{x_{0} \rightarrow M+0^{-}} F\left(x_{0}\right)=\lim _{x_{0} \rightarrow M+0^{+}} F\left(x_{0}\right) \\
\frac{d F}{d x_{0}}=\exp \left(\frac{2 \pi \delta_{1}}{\omega_{1}}\right)>1 \quad\left[M<x_{0} \leq F^{2}(M)\right] \\
\lim _{x_{0} \rightarrow M+0^{-}} \frac{d F}{d x_{0}}=-\infty \quad\left[\omega_{1} \tau_{d} \neq \theta\right] .
\end{gathered}
$$

Eq.(8) shows that the value of $F$ is continuous at $M$. Eqs.(9) and (10) show that the differential coefficient of $F$ is discontinuous at $M$.

In the following discussions, we assume that the following condition is satisfied:

$$
\frac{d^{2} F}{d x_{0}^{2}}>0 \quad\left[S<x_{0}<M\right] .
$$

This assumption is verified by computer calculations.

Then, if

$$
\left.\frac{d F}{d x_{0}}\right|_{x_{0}=F(M)}<-1
$$

is satisfied, Eq.(7) is satisfied. Therefore we regard Eq.(12) as the chaos generating condition. Fig. 6 shows the chaos generating region in two-parameter space.

Fig. 7 shows one-parameter bifurcation diagram.

Similarly, it can be proved that the circuit- 2 exhibits chaos in the sense of theorem-1.

Lastly some of the typical experimental results and the corresponding simulated results are shown in Fig. 8 . In these experiments, $\delta_{1}$ and $\tau_{d}$ are fixed, and $\delta_{2}$ is used as a parameter. Experimental results match the theoretical ones very well both qualitatively and quantitatively.

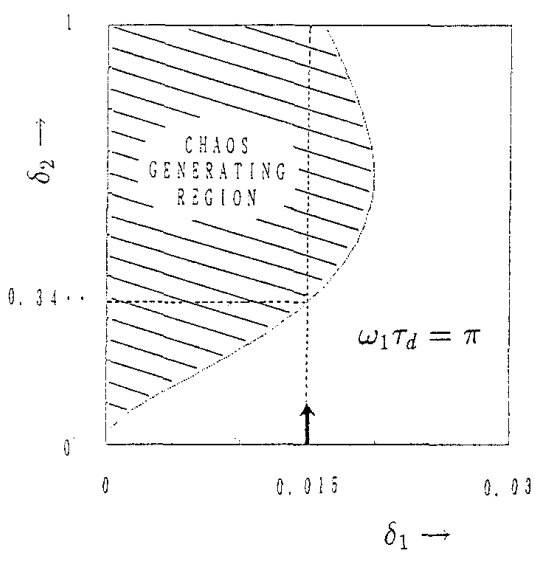

Fig.6 Chaos generating region $\left(\omega_{1} \tau_{d}=\pi\right)$ 


\section{CONCLUSIONS}

We have proposed two types of self-oscillatory circuits containing time delay which exhibit chaos and have analyzed them strictly. By using mapping method, we could derive the 1-dimensional Poincaré map explicitly from each circuit, and the Poincaré map was proved to have a positive Liapunov number with computer assistances. Moreover the theoretical results were verified by circuit experiments.

\section{REFERENCES}

[1] T. Maruyama, N. Inaba, Y. Nishio and S. Mori: "Chaos in an Auto Gain Controlled Oscillator Containing Time Delay", Trans. IEICE, vol. J 72-A, pp.1814-1820, Nov. 1989

Fig.7 1-parameter bifurcation diagram $\left(\delta_{1}=0.015, \omega_{1} \tau_{d}=\pi\right)$
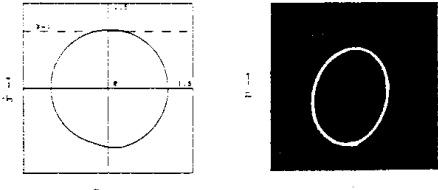

Fic. ¿-j Periodic solution with period

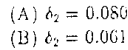
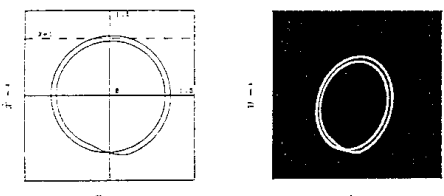

Fig. 8 -2 Periodic solution with period 2 (A) $\delta_{y}=0.140$ (B) $\delta_{2}=0.12$

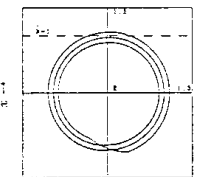

$=-$

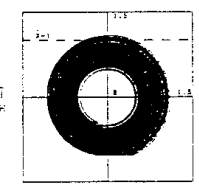

(A)

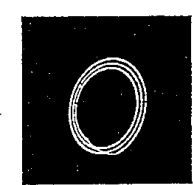

i-

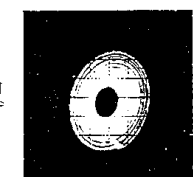

-

(B)

Fig.8 Experimental results(B) and corresponding simulated results(A)

$\left(\delta_{1}=0.015, \omega_{1} \tau_{d}=\pi\right)$

[2] Y. Inoue and Y. Ueda: "Self-Modulation Phenomena in a Self-Oscillatory System Containing Dead Time", Trans. IEICE, vol. J 64-A, pp.791-792, Sep. 1981.

[3] A. Kouda and S. Mori : "Oscillations in a Self-Oscillatory System Containing Time Delay", Trans. IEICE, vol. J 72-A, pp.309-318, Feb. 1989.

[4] A. Lasota and James A.Yorke: "On the existence of invariant measures for piecewise monotonic transformations", Trans. Amer. Math. Soc., vol.186, pp.481-488, Dec. 1973.

[5] Tien-Yien Li and James A.Yorke: "Ergodic transformations from an interval into itself", Trans. Amer. Math. Soc., vol.235, pp.183-192, Jan. 1978. 